\title{
Three-dimensional photoacoustic and ultrasonic endoscopic imaging of two rabbit esophagi
}

Joon-Mo Yang, Christopher P. Favazza, Junjie Yao, Ruimin Chen, Qifa Zhou, et al.

Joon-Mo Yang, Christopher P. Favazza, Junjie Yao, Ruimin Chen, Qifa Zhou, K. Kirk Shung, Lihong V. Wang, "Three-dimensional photoacoustic and ultrasonic endoscopic imaging of two rabbit esophagi," Proc. SPIE 9323, Photons Plus Ultrasound: Imaging and Sensing 2015, 932334 (11 March 2015); doi: 10.1117/12.2080294

SPIE. Event: SPIE BiOS, 2015, San Francisco, California, United States 


\title{
Three-dimensional photoacoustic and ultrasonic endoscopic imaging of two rabbit esophagi
}

\author{
Joon-Mo Yang ${ }^{1,4}$, Christopher Favazza ${ }^{1,3,4}$, Junjie Yao ${ }^{1}$, Ruimin Chen $^{2}$, Qifa Zhou ${ }^{2}$, K. Kirk Shung ${ }^{2}$, \\ and Lihong V. Wang ${ }^{1 *}$ \\ ${ }^{1}$ Optical Imaging Laboratory, Department of Biomedical Engineering, Washington University in St. \\ Louis, One Brookings Drive, Campus Box 1097, St. Louis, Missouri 63130, USA \\ ${ }^{2}$ Ultrasonic Transducer Resource Center, Department of Biomedical Engineering, University of \\ Southern California, 1042 Downey Way, University Park, Los Angeles, California 90089, USA \\ ${ }^{3}$ Current affiliation: Department of Radiology, Mayo Clinic, Rochester, Minnesota, USA
}

\begin{abstract}
The addition of photoacoustic endoscopy to conventional endoscopic ultrasound offers imaging capabilities that may improve diagnosis and clinical care of gastrointestinal tract diseases. In this study, using a 3.8-mm diameter dual-mode photoacoustic and ultrasonic endoscopic probe, we investigated photoacoustic and ultrasonic image features of rabbit esophagi. Specifically, we performed ex vivo imaging of intact rabbit esophagi and correlated the acquired images with histology. Without motion artifact-based limitations, we were able to utilize the full resolving power of the endoscopic device and acquire the first three-dimensional vasculature map of the esophagus and mediastinum, along with coregistered tissue density information. Here, we present the experimental results and discuss potential clinical applications of the technique.
\end{abstract}

Keywords: Photoacoustic endoscopy, endoscopic ultrasound, upper gastrointestinal tract, three-dimensional imaging, rabbit esophagus, rabbit mediastinum, vasculature, Barrett's esophagus.

\section{INTRODUCTION}

Previously, we demonstrated the clinical potential of photoacoustic endoscopy (PAE) [1] by performing in vivo imaging of the upper gastrointestinal tracts of rabbits, using a 3.8-mm diameter photoacoustic endoscope [2,3]. In addition to the first in vivo demonstration, we also showed the complementary benefits of dual-mode photoacoustic (PA) and ultrasonic (US) endoscopy for the characterization of the anatomic structures in the mediastina. Among optical endoscopy techniques [4-15], our PAE system [2,3] provided the first three-dimensional visualization of several major organs in the mediastina, such as the lung and trachea, and the neighboring blood vessels, such as the aorta and caudal vena cava. Thus, we demonstrated the deep transesophageal imaging capability of PAE. Further, it has been shown that PAE complements conventional endoscopic ultrasound (EUS) [16-18] by providing detailed images of vascular structures based on its unique optical absorption contrast and also by providing functional information, such as oxygen saturation levels. However, in the previous study [2, 3], the acquired three-dimensional images suffered from respiratory motion artifacts, which required the application of surface-alignment and spatial filtering algorithms to clearly visualize the major organs around the esophagus. The strong respiratory motion artifacts precluded the visualization of threedimensional vasculature images of the esophageal wall, and the application of surface alignment and spatial filter algorithms greatly diminished the spatial resolution, thus blurring the image and negating their purpose. Clinically, the assessment of the esophagus is of great importance because it is associated with major pathologies of the upper gastrointestinal (GI) tract, such as Barrett's esophagus [19-21]. In recent years, there have been many reports on the potential for assessing esophageal vasculature for the diagnosis of upper GI tract related diseases [22-26].

${ }^{4}$ These authors contributed equally to this work. "Corresponding author: 1hwang@biomed.wustl.edu

Photons Plus Ultrasound: Imaging and Sensing 2015, edited by Alexander A. Oraevsky, Lihong V. Wang

Proc. of SPIE Vol. 9323, 932334 - (c) 2015 SPIE · CCC code: 1605-7422/15/\$18

doi: $10.1117 / 12.2080294$

Proc. of SPIE Vol. $9323932334-1$ 
Using the 3.8-mm diameter dual-mode PAE and EUS imaging probe, we performed ex vivo imaging of two intact rabbit esophagi and neighboring regions without the effects of motion artifacts and correlated the acquired images with histology. Such an ex vivo imaging strategy is commonly employed when evaluating the capability of new technologies to image anatomical features for the first time $[10,13]$. Utilizing the full resolving power of the endoscopic device, we acquired the first in situ three-dimensional vasculature maps of the esophagi and mediastina, along with co-registered tissue density information (at present, to the best of our knowledge, no other reported photoacoustic endoscopic systems are capable of volumetric intra-luminal imaging in situ). Blood vessels with apparent diameters as small as $\sim 190 \mu \mathrm{m}$ were photoacoustically resolved without the aid of any exogenous contrast agents. More importantly, with the dualmodal PA and US imaging capability of the system, we were able to better identify and characterize the detailed anatomic structures of the esophageal lumen, such as the mucosal and submucosal layers in the esophageal wall. In this paper, we further discuss the potential clinical applications of our endoscopic technique and consider related technical issues to be resolved before full clinical use.

\section{MATERIALS AND METHODS}

\subsection{8-mm diameter dual-mode PAE and EUS imaging probe and its peripheral systems}

Figure 1 shows the 3.8-mm diameter probe and peripheral systems utilized for this ex vivo imaging study. The system can provide co-registered PA and US images with axial resolutions of $\sim 80 \mu \mathrm{m}$ and $\sim 60 \mu \mathrm{m}$ and maximum transverse resolutions of $\sim 55 \mu \mathrm{m}$ and $\sim 30 \mu \mathrm{m}$, respectively. The radial dependences of the transverse resolutions are available in the previous report [2]. Both the PA and US resolution are determined by the acoustic parameters of the employed focused US transducer $\left(\mathrm{LiNbO}_{3}, \sim 36 \mathrm{MHz}, 65 \%\right.$ fractional bandwidth). PA imaging was conducted using a nanosecond pulsed laser system consisting of a tunable dye laser (Cobra HRR, Sirah), pumped by a solid-state, diode-pumped Nd:YLF laser (INNOSLAB IS811-E, EdgeWave), which generated $584 \mathrm{~nm}$ laser illumination, with a $\sim 10$ ns pulse width and $\sim 0.3 \mathrm{~mJ}$ pulse energy. For US imaging, we employed an US pulser-receiver (5072PR, Panametrics) which provided sharp electric pulses to the US transducer to generate acoustic pulses. All the US and PA signals detected by the transducer were also amplified by the same US pulser-receiver and digitally recorded by the DAQ card (200 MHz, 12 bit, NI PCI-5124, National Instruments).

We acquired PA and US cross-sectional images with a frame rate of $\sim 4 \mathrm{~Hz}$ for each imaging mode, which was the maximum frame rate, determined by the mechanical capacity of the employed micromotor [2]. With the set laser energy and wavelength, the endoscope's maximal radial imaging depth was $\sim 7 \mathrm{~mm}$ from the endoscope's surface, and the angular field-of-view (FOV) was $\sim 270^{\circ}$. A $90^{\circ}$ region of the endoscope was blocked by the stainless steel housing of the probe. More detailed information on the system is available in our previous report $[2,3]$.

(a)

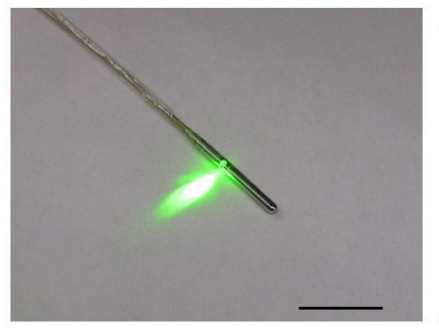

(b)

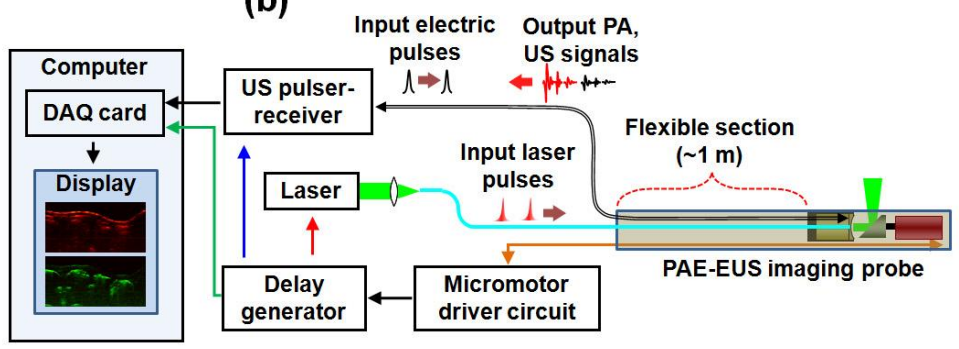

Figure 1. (a) Photo of the 3.8-mm diameter PA endoscopic probe. The inner cavity of the endoscope is filled with deionized water to provide acoustic coupling between the probe's imaging window and the US transducer. Scale bars, $3 \mathrm{~cm}$.

(b) Block diagram showing the endoscope and peripheral systems.

\subsection{Animal experiment}

With the endoscopic system, we imaged the intact esophagi of two adult New Zealand white rabbits ( $\sim \mathrm{kg}, \sim 6$-monthold, Myrtle's Rabbitry) ex vivo. To reduce the likelihood of ingesta in the upper GI tracts, we fasted the rabbits beginning $\sim 12 \mathrm{hr}$ before the experiments. Prior to endoscopic imaging, we euthanized the rabbit by an overdose of sodium pentobarbital $(150 \mathrm{mg} / \mathrm{kg})$ injected in the marginal ear vein, then we placed the rabbit on an inclined stage 
$\left(\sim 10^{\circ}\right)$ in the supine position. Just before probe insertion, we filled the esophagus with water using an enteral feeding syringe connected to a rubber feeding tube to provide acoustic coupling between the endoscope and the tissue. Afterwards, we immediately inserted the endoscopic probe through the mouth and advanced it approximately $25-30 \mathrm{~cm}$, to the point at which the probe could no longer be gently advanced, and then we started to record co-registered PAEEUS images. During image acquisition, the probe was slowly and mechanically pulled out of the esophagus over a $\sim 12$ $\mathrm{cm}$ range, using a motorized translation stage at a speed of $\sim 160 \mu \mathrm{m} / \mathrm{s}$. About 3000 B-scan slices with a longitudinal spacing of $\sim 40 \mu \mathrm{m}$ were acquired for each imaging mode. After finishing the imaging experiment, we dissected the animal and collected samples from the esophagus for histological analysis, which were compared with PA and US images. All procedures in the animal experiments followed protocols approved by the Institutional Animal Care and Use Committee at Washington University in St. Louis.

\section{RESULTS}

In Figure 2, we present two sets of co-registered PAE and EUS radial-maximum amplitude projection (RMAP) images acquired from the esophagi and mediastina of the two rabbits. Each animal's RMAP images were processed from an original three-dimensional data set covering a $\sim 18 \mathrm{~mm}$ diameter and $\sim 12 \mathrm{~cm}$ long cylindrical volume. To more clearly display the vasculature differences, we separated the esophageal and mediastinal images based on the thicknesses of the esophagus wall $(\sim 400 \mu \mathrm{m})$. This image separation was conducted after Hilbert transforming to the raw data and extracting the envelope of the bipolar signal. The PA-only images represent light absorption distributions at the $584 \mathrm{~nm}$ laser wavelength. At this laser wavelength, the PA signal is sensitive only to the total hemoglobin concentration. The US images represent the echogenicity distribution of the imaged structures, in the same way as conventional pulse-echo imaging detects acoustic waves reflected from target tissue.

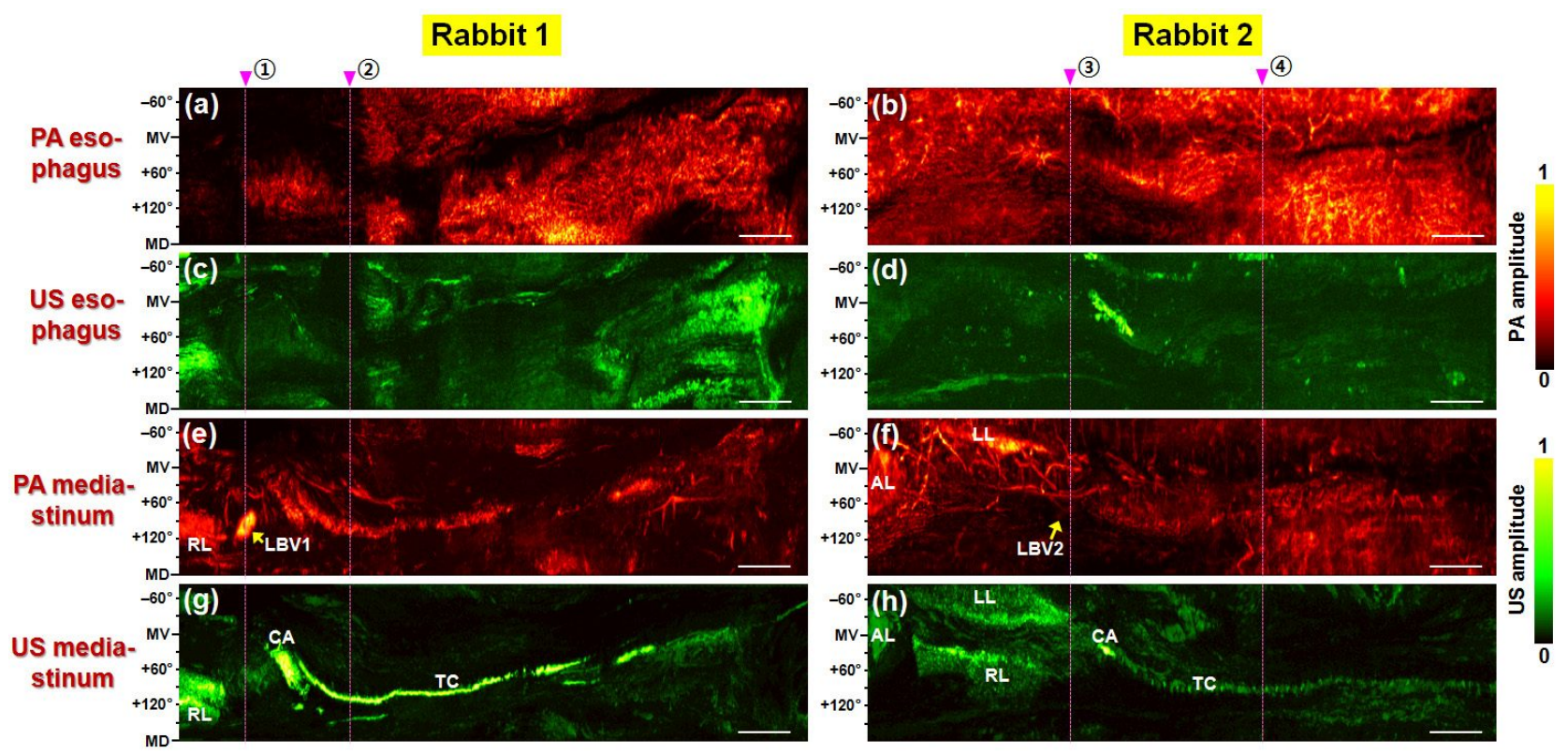

Figure 2. (a, b) Normalized PA-RMAP images of two rabbit esophagi, showing the total hemoglobin distribution (views from the inside of the esophagus). In each image, the left- and right-hand sides correspond to the lower and upper esophagus, respectively, and the imaged area covers an $\sim 270^{\circ}$ angular FOV (vertical) and an $\sim 12 \mathrm{~cm}$ long pullback distance (horizontal). (c, d) Corresponding normalized US-RMAP images of the PA-RMAP images shown in (a) and (b), respectively. (e, f) Normalized PA-RMAP images of the mediastina for the two rabbits, with the esophageal signals excluded during the RMAP construction. $(\mathrm{g}, \mathrm{h})$ Corresponding normalized US-RMAP images of the PA-RMAP images shown in (e) and (f), respectively. AL, accessory lobe; LL, left lobe; RL, right lobe of the lung; CA, carina; TC, trachea; LBV, large blood vessel. In (a)-(h), the approximate mid-ventral (MV) position and angular displacement from the MV position are marked along the vertical $\varphi$-axis; the positive and negative values correspond to the right and left sides of the animal, and MD denotes the mid-dorsal position. All PA and US signal amplitudes are mapped on a linear scale. Scale bars, $1 \mathrm{~cm}$ (horizontal only). 
As shown in the esophageal PA-RMAP images [Figs. 2(a) and 2(b)], PAE provided clear views of the vasculatures distributed in the walls of the esophagi. The PAE images show that the two rabbits possess quite different vascular structures over the imaged esophageal regions. Additionally from the three-dimensional PA images, we could begin to map vascular connections between the esophagus and the exterior organs distributed in the mediastina for the first time. In the case of the mediastinal RMAP images [Figs. 2(e) and 2(f)], we could detect PA signals from several major organs, such as the accessory lobe (AL), the left lobe (LL), the right lobe (RL) of the lung, the carina (CA), and the trachea (TC), distributed in the mediastinal regions, as with our previous in vivo study [2]. However, in the ex vivo images, we could visualize the peripheral vasculature details, with the full resolving power of the endoscopic system an apparent vessel as small as $190 \mu \mathrm{m}$ was resolved.

One interesting set of structures newly-found in this study can be seen in the regions marked with the yellow arrows (i.e., near the carina) in the PA mediastinal RMAP images [Figs. 2(e) and 2(f)]. These structures appeared in multiple image data sets from the same rabbit, and they were distributed within $\sim 1 \mathrm{~mm}$ from the outer wall of the esophagus (see Figures 3(a) and 3(c)). Since they generated strong PA signals, it is obvious that they are highly light-absorbing structures. However, corresponding US images [Figs. 2(g) and 2(h)] did not show high echoic responses comparable to the magnitudes of PA signals. Considering these two image features, these structures are likely blood vessels. After the post-experiment surgical examinations of the corresponding locations, we could identify that the imaged structures are a part of the pulmonary vascular system (Similar structures were also reported in our recent paper [27]). Monitoring the oxygen saturation level in the pulmonary vein is an important issue in many surgical procedures, and the presented capability of the PAE system suggests its potential for this important application.

In Figure 3, we present two sets of cross-sectional images [Figs. 3(a)-3(1)] selected at the marked positions (dashed lines) in Figure 2, along with two histologic images [Figs. 3(m) and 3(n)] of samples harvested from the mid-esophageal regions of the same animals. Since the esophageal lumens were less than $10 \mathrm{~mm}$ from the probe along the entire length of the esophagus, we cropped the presented PAE [Figs. 3(a)-3(d)], EUS [Figs. 3(e)-3(h)], and combined PAE-EUS [Figs. 3(i)-3(l)] cross-sectional images at $12 \mathrm{~mm}$ diameter imaging depths to more clearly display features near the esophageal walls.

Although the EUS images [Figs. 3(e)-3(h)] sometimes showed clear boundaries of the mucosal and submucosal layers [Fig. 3(f)], overall US signal intensities of those layers were very weak [Figs. 3(e), 3(g), and 3(h)] because we employed a focused US transducer and set the gain (dynamic range) of the signal amplifier to optimize registration of signals from other hyper-echoic regions, such as the trachea. Thus, the inner boundary of the esophageal walls in the US images appeared strongly only when they were located near the focal point of the transducer and their surface was perpendicular to the acoustic axis. However, PAE images [Figs. 3(a)-3(d)] provided clear boundaries of the esophageal walls over the entire length of the esophagi (i.e., not only in the presented cross-sectional images) because many capillaries are densely distributed in the submucosal layers. Thus, based on the PA cross-sectional images [Figs. 3(a)$3(\mathrm{~d})$ ], we could estimate the wall thickness of the two esophagi to be $\sim 400-600 \mu \mathrm{m}$, values which are slightly thinner than those in the histologic images [Figs. 3(m) and 3(n)]. It is likely that the esophageal walls in the histologic images were thickened from unavoidable distortion that occurs during the histological fixation process. In addition to the clear delineation of the submucosal layers of the esophageal walls [Fig. 3(j)], the PAE images were advantageous for better identifying the major blood vessels surrounding the esophagi, such as the large blood vessels (LBV1 \& LBV2) which are assumed to be a part of the pulmonary vascular system [Figs. 3(a) and 3(c)]. 


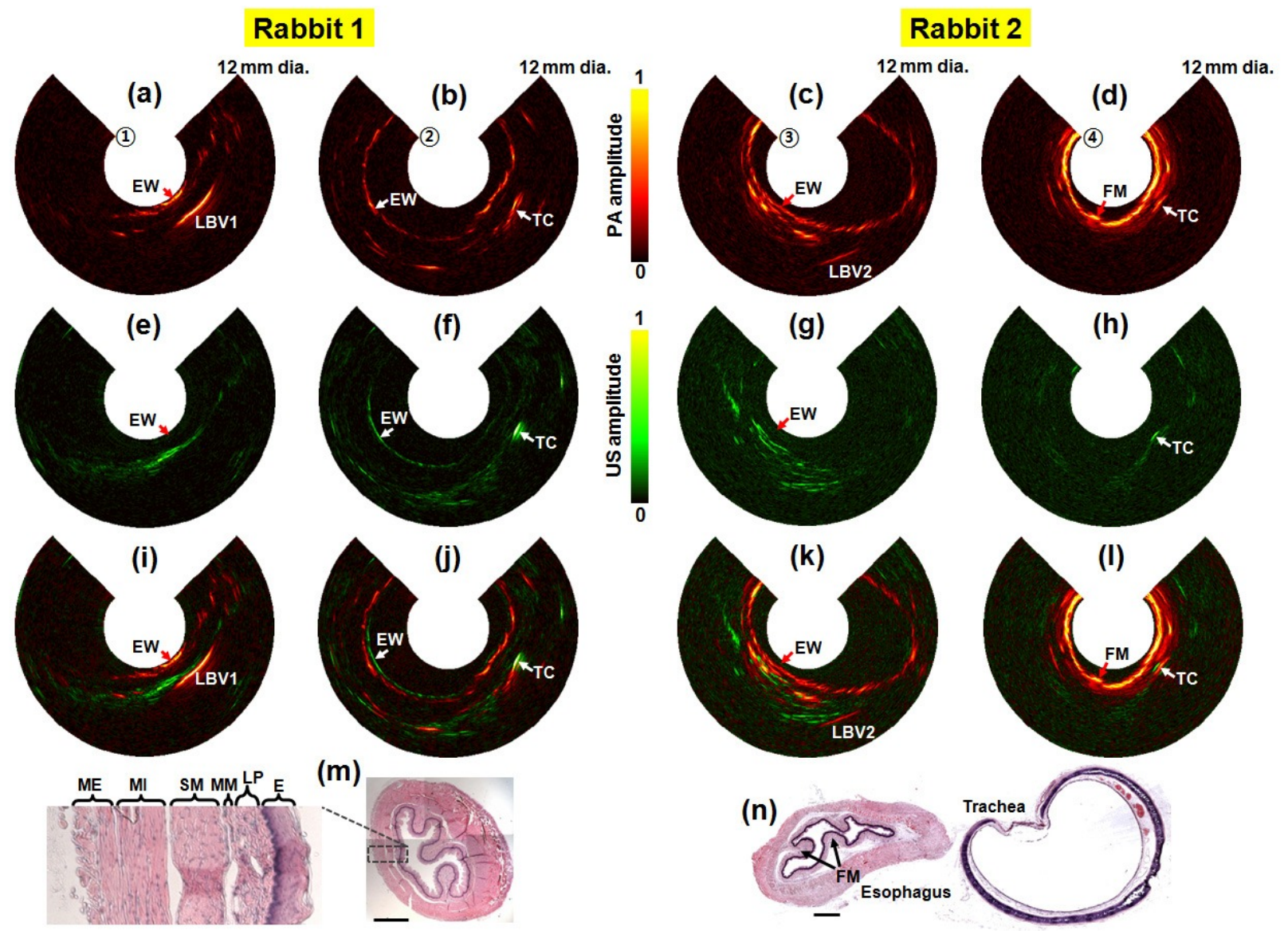

Figure 3. (a-d) PAE, (e-h) EUS, and (i-l) combined PAE-EUS B-scan images of the esophagi chosen from the marked positions. Each image covers a $12 \mathrm{~mm}$ diameter FOV (i.e., $\sim 4.1 \mathrm{~mm}$ in imaging depth). EW, esophageal wall; LBV1 \& LBV2, large blood vessels; TC: trachea. All PA and US signal amplitudes are mapped on a linear scale. (m, n) Histologic images (H\&E stain) of the two rabbit esophagi. E, stratified squamous epithelium; LP, lamina propria; MM, muscularis mucosa; SM, submocosa; MI, muscularis interna; ME, muscularis externa; FM, folded mucosa. Scale bars, $1 \mathrm{~mm}$.

\section{DISCUSSION}

As shown in the results section, the major benefit of PAE is that it can provide perilumenal vasculature images without the aid of contrast agents. US image contrasts for vascular imaging were not nearly as high as for PAE. To ultrasonically visualize blood vessels, EUS typically requires US-Doppler imaging or the use of contrast agents [1618]; however, both of these strategies possess drawbacks (i.e. accuracy, safety, etc.), which make them non-ideal solutions. PAE inherently employs endogenous contrast of vascular structures, which can promote better identification of anatomical structures. Thus, by providing images complementary to conventional EUS, PAE could potentially provide many clinical contributions in endoscopic imaging. In the case of upper GI tract disease diagnosis and treatment, PAE could guide various surgical procedures, such as esophagectomy [28-30], or help diagnose esophageal diseases, such as Barrett's esophagus [19-21].

To make this endoscopic technique more clinically applicable, several technical issues must be resolved. Improving the scanning speed is most important [31-34]. Development of array transducer-based electronic scanning endoscopic systems [35] would be a promising direction because such endoscopic systems could provide cross-sectional images with a single laser pulse and also would enable a high image frame rate. In addition to improving the scanning speed, 
increasing the spatial resolution is another important direction. In this study, we demonstrated acoustic-resolution PAE imaging, which provides endoscopic images with the spatial resolution determined by the acoustic parameters of the employed transducer. However, the lateral resolution could be greatly improved if an optical focusing method were applied, as we demonstrated in a recent work [36]. A third developmental goal is to increase the imaging depth of acoustic-resolution PAE system [37]. In this study, we could photoacoustically visualize several major blood vessels connected to the pulmonary and cardiovascular systems. However, improving the penetration depth could make this technique more powerful by providing structural and functional information about the major organs, such as the heart, complementing a current EUS-based technique [16-18] referred to as transesophageal echocardiography (TEE). In addition to these technical improvements, to fully understand the anatomic structural images provided by PAE, more systematic studies should be performed in concert with existing imaging modalities, such as X-ray computed tomography [38].

\section{ACKNOWLEDGMENT}

We thank Prof. James Ballard for his attentive reading of the manuscript. We also thank Lisa Andrews-Kaminsky,

Jenny Kalishman, and Suellen Greco for helping with the animal preparation, surgery, and image interpretation. This work was sponsored in part by National Institutes of Health grants EB016986 (NIH Director's Pioneer Award), R01 CA186567 (NIH Director's Transformative Research Award), and R01 CA159959. L.W. has a financial interest in Microphotoacoustics, Inc. and Endra, Inc., which, however, did not support this work.

\section{REFERENCES}

[1] Yang, J. M., Maslov, K., Yang, H. C., Zhou, Q., Shung, K. K. and Wang, L. V., "Photoacoustic endoscopy," Opt. Lett. 34(10), 1591-1593 (2009).

[2] Yang, J. M., Favazza, C., Chen, R., Yao, J., Cai, X., Maslov, K., Zhou, Q., Shung, K. K. and Wang, L. V., "Simultaneous functional photoacoustic and ultrasonic endoscopy of internal organs in vivo," Nat. Med. 18(8), 1297-1302 (2012).

[3] Yang, J. M., Favazza, C., Chen, R., Yao, J., Cai, X., Maslov, K., Zhou, Q., Shung, K. K. and Wang, L. V., "Toward dual-wavelength functional photoacoustic endoscopy: laser and peripheral optical systems development," Proc. SPIE 8223, 822316 (2012).

[4] Yun, S. H., Tearney, G. J., Vakoc, B. J., Shishkov, M., Oh, W. Y., Desjardins, A. E., Suter, M. J., Chan, R. C., Evans, J. A., Jang, I. K., Nishioka, N. S., De Boer, J. F. and Bouma, B. E., "Comprehensive volumetric optical microscopy in vivo," Nat. Med. 12(12), 1429-1433 (2006).

[5] Evans, J. A., Poneros, J. M., Bouma, B. E., Bressner, J., Halpern, E. F., Shishkov, M., Lauwers, G. Y., MinoKenudson, M., Nishioka, N. S. and Tearney, G. J., "Optical coherence tomography to identify intramucosal carcinoma and high-grade dysplasia in Barrett's esophagus," Clin. Gastroenterol. Hepatol. 4(1), 38-43 (2006).

[6] Chen, Y., Aguirre, A. D., Hsiung, P. L., Desai, S., Herz, P. R., Pedrosa, M., Huang, Q., Figueiredo, M., Huang, S. W., Koski, A., Schmitt, J. M., Fujimoto, J. G. and Mashimo, H., "Ultrahigh resolution optical coherence tomography of Barrett's esophagus: preliminary descriptive clinical study correlating images with histology," Endoscopy 39(7), 599-605 (2007).

[7] Evans, J. A., Bouma, B. E., Bressner, J., Shishkov, M., Lauwers, G. Y., Mino-Kenudson, M., Nishioka, N. S. and Tearney, G. J., "Identifying intestinal metaplasia at the squamocolumnar junction by using optical coherence tomography," Gastrointest. Endosc. 65(1), 50-56 (2007).

[8] Vakoc, B. J., Shishko, M., Yun, S. H., Oh, W. Y., Suter, M. J., Desjardins, A. E., Evans, J. A., Nishioka, N. S., Tearney, G. J. and Bouma, B. E. "Comprehensive esophageal microscopy by using optical frequency-domain imaging (with video)," Gastrointest. Endosc. 65(6), 898-905 (2007).

[9] Suter, M. J., Vakoc, B. J., Yachimski, P. S., Shishkov, M., Lauwers, G. Y., Mino-Kenudson, M., Bouma, B. E., Nishioka, N. S. and Tearney, G. J., "Comprehensive microscopy of the esophagus in human patients with optical frequency domain imaging," Gastrointest. Endosc. 68(4), 745-753 (2008).

[10] Cobb, M. J., Hwang, J. H., Upton, M. P., Chen, Y., Oelschlager, B. K., Wood, D. E., Kimmey, M.. B. and Li, X., "Imaging of subsquamous Barrett's epithelium with ultrahigh-resolution optical coherence tomography: a histologic correlation study," Gastrointest. Endosc. 71(2), 223-730 (2010). 
[11] Gora, M. J., Sauk, J. S., Carruth, R. W., Gallagher, K. A., Suter, M. J., Nishioka, N. S., Kava, L. E., Rosenberg, M., Bouma, B. E. and Tearney, G. J., "Tethered capsule endomicroscopy enables less invasive imaging of gastrointestinal tract microstructure," Nat. Med. 19(2), 238-240 (2013).

[12] Kiesslich, R., Gossner, L., Goetz, M., Dahlmann, A., Vieth, M., Stolte, M., Hoffman, A., Jung, M., Nafe, B., Galle, P. R. and Neurath, M. F., "In vivo histology of Barrett's esophagus and associated neoplasia by confocal laser endomicroscopy," Clin. Gastroenterol. Hepatol. 4(8), 979-987 (2006).

[13] Kang, D., Suter, M. J., Boudoux, C., Yoo, H., Yachimski, P. S., Puricelli, W. P., Nishioka, N. S., Mino-Kenudson, M., Lauwers, G. Y., Bouma, B. E. and Tearney, G. J., "Comprehensive imaging of gastroesophageal biopsy samples by spectrally encoded confocal microscopy," Gastrointest Endosc. 71(1), 35-43 (2010).

[14] Qiu, L., Pleskow, D. K., Chuttani, R., Vitkin, E., Leyden, J., Ozden, N., Itani, S., Guo, L., Sacks, A., Goldsmith, J. D., Modell, M. D., Hanlon, E. B., Itzkan, I. and Perelman, L. T., "Multispectral scanning during endoscopy guides biopsy of dysplasia in Barrett's esophagus," Nat. Med. 16, 603-606 (2010).

[15] Terry, N. G., Zhu, Y., Rinehart, M. T., Brown, W. J., Gebhart, SC, Bright, S., Carretta, E., Ziefle, C. G., Panjehpour, M., Galanko, J., Madanick, R. D., Dellon, E. S., Trembath, D., Bennett, A., Goldblum, J. R., Overholt, B. F., Woosley, J. T., Shaheen, N. J. and Wax, A., "Detection of dysplasia in Barrett's esophagus with in vivo depth-resolved nuclear morphology measurements," Gastroenterology 140, 42-50 (2011).

[16] Dietrich, C., [Endoscopic Ultrasound: An Introductory Manual and Atlas], Thieme, New York (2011).

[17] Hawes, R. H. and Fockens, P., [Endosonography], Saunders Elsevier, Canada (2006).

[18] Shami, V. M. and Kahaleh, M., [Endoscopic Ultrasound], Humana Press, New York (2010).

[19] Spechler, S. J., Sharma, P., Souza, R. F., Inadomi, J. M. and Shaheen, N. J., "American Gastroenterological Association Technical Review on the Management of Barrett's Esophagus," Gastroenterology 140(3), e18-e52 (2011).

[20] Wilson, B. C., "Detection and treatment of dysplasia in Barrett's esophagus: a pivotal challenge in translating biophotonics from bench to bedside," J. Biomed. Opt. 12(5), 051401 (2007).

[21] Sato, F., Jin, Z., Schulmann, K., Wang, J., Greenwald, B. D., Ito, T., Kan, T., Hamilton, J. P., Yang, J., Paun, B., David, S., Olaru, A., Cheng, Y., Mori, Y., Abraham, J. M., Yfantis, H. G., Wu, T. T., Fredericksen, M. B., Wang, K. K., Canto, M., Romero, Y., Feng, Z. and Meltzer, S. J, "Three-tiered risk stratification model to predict progression in Barrett's esophagus using epigenetic and clinical features," PLoS One. 3(4), e1890 (2008).

[22] Kara, M. A., Ennahachi, M., Fockens, P., ten Kate, F. J. and Bergman, J. J, "Detection and classification of the mucosal and vascular patterns (mucosal morphology) in Barrett's esophagus by using narrow band imaging," Gastrointest. Endosc. 64(2), 155-166 (2006).

[23] Singh, R., Anagnostopoulos, G. K., Yao, K., Karageorgiou, H., Fortun, P. J., Shonde, A., Garsed, K., Kaye, P. V., Hawkey, C. J. and Ragunath, K., "Narrow-band imaging with magnification in Barrett's esophagus: validation of a simplified grading system of mucosal morphology patterns against histology," Endoscopy 40(6), 457-463 (2008).

[24] Lee, M. M. and Enns, R., "Narrow band imaging in gastroesophageal reflux disease and Barrett's esophagus," Can. J. Gastroenterol. 23(2), 84-87 (2009).

[25] Thekkek, N., Pierce, M. C., Lee, M. H., Polydorides, A. D., Flores, R. M., Anandasabapathy, S. and RichardsKortum, R. R., "Modular video endoscopy for in vivo cross-polarized and vital-dye fluorescence imaging of Barrett's-associated neoplasia," J. Biomed. Opt. 18(2), 26007 (2013).

[26] Becker, V., Vieth, M., Bajbouj, M., Schmid, R. M. and Meining, A., "Confocal laser scanning fluorescence microscopy for in vivo determination of microvessel density in Barrett's esophagus," Endoscopy 40(11), 888-891 (2008).

[27] Yang, J. M., Favazza, C., Chen, R., Yao, J., Zhou, Q., Shung, K. K. and Wang, L. V., "Three-dimensional photoacoustic endoscopic imaging of the rabbit esophagus," PloS One 10, 00 (2015).

[28] Liebermann-Meffert, D. M., Luescher, U., Neff, U., Ruedi, T. P. and Allgower, M., "Esophagectomy without thoracotomy: is there a risk of intramediastinal bleeding? A study on blood supply of the esophagus," Ann. Surg. 206(2), 184-192 (1987).

[29] Luketich, J. D., Alvelo-Rivera, M., Buenaventura, P. O., Christie, N. A., McCaughan, J. S., Litle, V. R., Schauer, P.R., Close, J. M. and Fernando, H. C., "Minimally invasive esophagectomy: outcomes in 222 patients," Ann. Surg. 238(4), 486-494 (2003).

[30] Palanivelu, C., Prakash, A., Senthilkumar, R., Senthilnathan, P., Parthasarathi, R., Rajan, P. S. and Venkatachlam S., "Minimally invasive esophagectomy: thoracoscopic mobilization of the esophagus and mediastinal lymphadenectomy in prone position--experience of 130 patients," J. Am. Coll. Surg. 203(1), 7-16 (2006). 
[31] Favazza, C. P., Jassim, O., Cornelius, L. A. and Wang, L. V., "In vivo photoacoustic microscopy of human cutaneous microvasculature and a nevus," J. Biomed. Opt. 16(1), 016015 (2011).

[32] Yao, J., Huang, C. H., Wang, L., Yang, J. M., Gao, L., Maslov, K. I., Zou, J. and Wang, L. V., "Wide-field fastscanning photoacoustic microscopy based on a water-immersible MEMS scanning mirror," J. Biomed. Opt. 17(8), 080505 (2012).

[33] Li, C., Yang, J. M., Chen, R., Yeh, C.-H., Zhu, L., Maslov, K., Zhou, Q., Shung, K. K. and Wang, L. V., "Urogenital photoacoustic endoscope," Opt. Lett. 39(6), 1473-1476 (2014).

[34] Yang, J. M., Li, C., Chen, R., Zhou, Q., Shung, K. K. and Wang, L. V., "Catheter-based photoacoustic endoscope," J. Biomed. Opt. 19(6), 066001 (2014).

[35] Tsyboulski, D., Conjusteau, A. and Oraevsky, A., "Dual modality optoacoustic and laser ultrasound endoscopy system," Proc. SPIE 8943, 89432S (2014).

[36] Yang, J. M., Li, C., Chen, R., Rao, B., Yao, J., Yeh, C. H., Danielli, A., Maslov, K., Zhou, Q., Shung, K. K. and Wang, L. V., "Optical-resolution photoacoustic endomicroscopy in vivo," Biomed. Opt. Express 6(3), 918-932 (2015).

[37] Kruger, R. A., Kuzmiak, C. M., Lam, R. B., Reinecke, D. R., Del Rio, S. P. and Steed, D., "Dedicated 3D photoacoustic breast imaging," Med. Phys. 40(11), 113301 (2013).

[38] Webb, A. G., [Introduction to biomedical imaging], John Wiley \& Sons, Inc., Hoboken (2002). 